\title{
Effects of sequences of sucrose reward magnitudes with short ITIs in rats
}

\author{
R. A. BURNS \\ University of South Florida, Tampa, Florida 33620
}

\begin{abstract}
Two experiments assessed the role of aftereffect learning in rats rewarded with sucrose solutions. In Experiment 1, rats were trained in a single straight runway for two trials on each of 18 days, each trial terminating with either large $(20 \%$ scurose) or small (3\% sucrose) reward. The ITI was 3-5 min. The sequence of daily rewards for each of four groups was small-small (SS), small-large, (SL), large-small (LS), or large-large (LL). Response patterning and a simultaneous negative contrast effect were observed in LS and SL relative to the consistently rewarded controls. During 10 massed extinction trials, resistance to extinction was greatest for Group SL, followed in order by Groups SS, LL, and LS. Experiment 2 examined single alternation of large and small rewards administered for 10 trials on each of 31 days with an ITI of $60 \mathrm{sec}$. Reward for one group was $20 \%$ or $3 \%$ sucrose while another received 1 or 1045 -mg Noyes pellets. Appropriate patterning developed only in the food-pellet rewarded animals. The overall results suggest that sucrose rewards may produce highamplitude and long-duration aftereffects which interfere with learning in designs employing several massed daily trials, but which may facilitate learning-relative to food-pellet rewards-with longer intertrial intervals and fewer daily trials.
\end{abstract}

The performance of rats is often disrupted when reward magnitude is reduced (Crespi, 1942; Elliott, 1928). When it occurs, the disruption, currently termed the successive negative contrast effect (SuNCE), carries the implication that the S-R reinforcement principle (Hull, 1943) is at least an incomplete account of the role of reward in learning (Hull, 1952). But SuNCE does not always occur, and the importance of the SuNCE underscores the significance of exceptions to its occurrence. Mackintosh (1974) has organized these exceptions into three categories. ${ }^{1}$ First, the species of the experimental subject influences whether or not the effect can be obtained. Goldfish, for example, do not show the SuNCE (Gonzalez, Ferry, \& Powers, 1974; Gonzalez, Potts, Pitcoff, \& Bitterman, 1972; Lowes \& Bitterman, 1967; Mackintosh, 1971); nor do turtles (Pert \& Bitterman, 1970; Pert \& Gonzalez, 1974). Second, with increases in the delay of reward, within limits, the SuNCE may not occur in rats (Mackintosh \& Lord, 1973). Third, the SuNCE does not occur when different concentrations of sucrose are used as reward instead of different amounts of solid food (Flaherty, Riley, \& Spear, 1973; Homzie \& Ross, 1962; Rosen, 1966; Rosen \& Ison, 1965; Shanab, France, \& Young, 1975). Further effort is needed to delineate the processes which mediate contrast

This research is part of a doctoral dissertation supervised by H. D. Kimmel and submitted to the Department of Psychology at the University of South Florida. Portions of this paper were presented at the Annual Meeting of the Southern Society for Philosophy and Psychology, Atlanta, 1976. Requests for reprints should be sent to R. A. Burns, Charles L. Mix Memorial Fund, Georgia Southwestern College, Americus, Georgia 31709. effects when they occur and to understand the conditions under which contrast does not occur.

\section{EXPERIMENT 1}

This experiment was designed to examine a notion expressed first by Capaldi (1967) and later by Likely, Little, and Mackintosh (1971), Mackintosh (1974), and Likely (Note 1) concerning sucrose rewards. Capaldi's theory interprets the SuNCE in terms of generalization decrement (Capaldi \& Lynch, 1967). Each reward magnitude is assumed to produce specific reward stimuli (traces or aftereffects) which carry over from trial to trial, thereby forming a part of the stimulus complex which becomes conditioned to the instrumental response by reward. Reward reduction should alter this complex, temporarily disrupting performance. Capaldi (1967) suggested that sucrose rewards suffer from low responsivitythe degree to which the aftereffect of trial N-1 is present, to the exclusion of stimuli from other trial outcomes, on trial N. Likely (Note 1) later proposed that sucrose aftereffects are less effective, less persistent, and less discriminable than food-pellet aftereffects, thus reducing the probability of the SuNCE. The ad hoc status of this idea may be remedied if it generates testable predictions which differ for highand low-responsivity aftereffects. Simply adding to the list of failures to find contrast with sucrose, however, does nothing to clarify the role of its aftereffects, because, attractive as it may seem, the role of generalization decrement in the SuNCE is equivocal (e.g., Gonzalez, Ferry, \& Powers, 1974).

The present experiment sought to determine the 
effectiveness of sucrose aftereffects in an experimental arrangement first employed by Leonard (1969). Leonard's design, as opposed to the contrast design, clearly isolated the influence of aftereffect learning with food reward. Rats were given two training trials each day with large reward (24 pellets) on both trials (LL), small reward (2 pellets) on both trials (SS), or large reward on one trial and small reward on the other (LS or SL). The major evidence for aftereffect learning was that, of the four groups, SL was most resistant to extinction and LS was least resistant. A large reward preceding a small reward should result in conditioning the instrumental response, by small reward, to the aftereffect of large reward (SRL). Similarly, in Group SL the aftereffect of small reward (SRS) is conditioned to the response by large reward. When reward aftereffects are viewed on a stimulus continuum with large further from nonreward (SN) than small, extinction involves stimuli (SN) which are more similar to SRS than to SRL. Group SL, on this basis, has more generalized habit strength in extinction than Group LS.

If the failure of rats to show the SuNCE following sucrose reduction is due to the impoverished, nondifferentiable aftereffects of sucrose, then the ordering in extinction of groups given large and small sucrose rewards in sequence would be in marked contrast to that reported by Leonard (1969) with food pellets. The ordering should reflect the reward strength of sucrose but not the conditioning of its aftereffects to the response.

\section{Method \\ Subjects. The subjects were 32 experimentally naive male Long-Evans rats, approximately 90 days old at the beginning of preliminary training. The rats were secured from the animal colony maintained by the University of South Florida, Tampa,} Florida.

Apparatus. The apparatus was a wooden runway, $15.2 \mathrm{~cm}$ high, with a hinged clear Plexiglas cover. The startbox $(20.3 \times$ $15.2 \mathrm{~cm})$ and alley $(182.9 \times 10.2 \mathrm{~cm})$ were painted black. The goalbox $(30.5 \mathrm{~cm} \times 15.2 \mathrm{~cm})$ was painted white and was positioned at a $90^{\circ}$ angle to the end of the alley (entered by a right turn). Manually operated start and retrace doors were positioned at the beginning of the alley and $30.5 \mathrm{~cm}$ before the goalbox, respectively. Two photocells, one $7.6 \mathrm{~cm}$ beyond the first door and the other $15.2 \mathrm{~cm}$ before the goalbox, were located in one wall of the runway. Opening of the first door operated a microswitch and started the first of two Standard Electric timers. Interruption of the first light beam by the rat stopped the first timer and started the second timer, which was stopped by interruption of the second beam, thus allowing measurement of start and running times in units of $.01 \mathrm{sec}$.

Preliminary training. Animals were housed in individual cages with free access to food and water. The cages were located in the experimental room, which was lighted by two 75-W overhead bulbs on a controlled cycle of $8 \mathrm{~h}$ light and $16 \mathrm{~h}$ dark. Animals were placed on a walled exercise table for $20 \mathrm{~min}$ on each of the first 10 days. Beginning on Day 4, each animal was systematically reduced to $85 \%$ of its mean ad-lib body weight by controlling the amount of food given at approximately 22 -h intervals. Following weight reduction, the rats were randomly assigned to one of four groups-SS, LS, SL, or LL-where S represents small reward, L represents large reward, and the sequence of letters represents the order of delivery of rewards on the two daily acquisition trials. On Days 11 and 12, Groups LL and SS were placed on the table and allowed $15 \mathrm{~min}$ free access to $20 \%$ or $3 \%$ sucrose solution, respectively. Groups SL and LS were allowed $71 / 2 \mathrm{~min}$ access to each concentration in the appropriate order. The sucrose was mixed by percentage weight in tap water, and delivered in a glass coaster. Goalbox placements, administered on Days 13,14, and 15, consisted of placing each rat in the goalbox containing $1 \mathrm{ml}$ of sucrose solution. There were two placements each day with the concentration and sequence of rewards the same as that employed during the table feeding.

Acquisition training. There were 18 days of acquisition, two trials each day. A trial began with the opening of the start door $3 \mathrm{sec}$ after the rat was placed in the startbox. After interruption of the second light beam, the retrace door was closed, and the rat was removed after the reward was consumed. The experiment was run in two replications of 15 and 17 rats each. Groups SL, $L L$, and LS contained 4 rats in each replication. Group SS contained 3 rats in the first replication and 5 rats in the second. One rat in Group $L L$ of the second replication was removed for failure to consume the reward on three successive trials. Throughout the experiment, subjects were run in squads so that the intertrial interval (ITI) was approximately 3-5 min. Subjects spent the ITI in the living cage with water available. The order in which the groups were run was SS, SL, LS, and LL in the first replication, and that order was reversed for the second replication. The reward was $1 \mathrm{ml}$ of either $20 \%$ or $3 \%$ sucrose. Approximately $15 \mathrm{~min}$ after the end of a daily running, each rat was weighed and fed its calculated ration in the home cage.

Extinction training. Two days of extinction, consisting of 10 nonreinforced trials per day, followed acquisition. The order of running by groups and rotation within groups remained the same as in acquisition. If a rat remained in any section of the runway for $60 \mathrm{sec}$, it was placed in the goalbox with the retrace door closed and a time of $60 \mathrm{sec}$ was recorded for that section and any remaining section. Subjects were removed from the goalbox after $30 \mathrm{sec}$.

\section{Results}

Extinction. All raw time scores were transformed $[10 \times \ln (x+1)]$, and separate analyses were performed on the start and run measures. The overall findings of the experiment are represented in Figure 1 , which shows the mean running times of the four groups (SS, SL, LS, and LL) in two-trial blocks for each of the 18 days of acquisition, and the 2 days of extinction. It is apparent in this figure that Groups LS and SL differed markedly in rate of extinction (SL was more persistent) despite their similar asymptotic acquisition performance, and Group LL extinguished faster than Group SS.

Unweighted means analyses of variance performed on each extinction day treated the difference between Trial 1 and Trial 2 magnitudes as a between-subject factor. The start measure was relatively insensitive, but analysis of run times showed that on Day 1 of extinction, both Trial 1 and Trial 2 magnitude effects were significant $[\mathrm{F}(1,27)=26.67, \quad \mathrm{p}<.01$; $F(1,27)=5.22, p<.05]$. Small reward on the first trial (Groups SL and SS) produced greater resistance to extinction than large (LS and LL), and large reward on the second trial (SL and LL) produced greater resistance than small (LS and SS). These 
Figure 1. Acquisition and extinction for each of the four groups (SS, SL, LS, and LL) in Experiment 1. Each point in acquisition represents the mean transformed run time of the 2 daily trials. The 20 trials of extinction are also in blocks of 2 trials and are separated for the $\mathbf{2}$ extinction days.
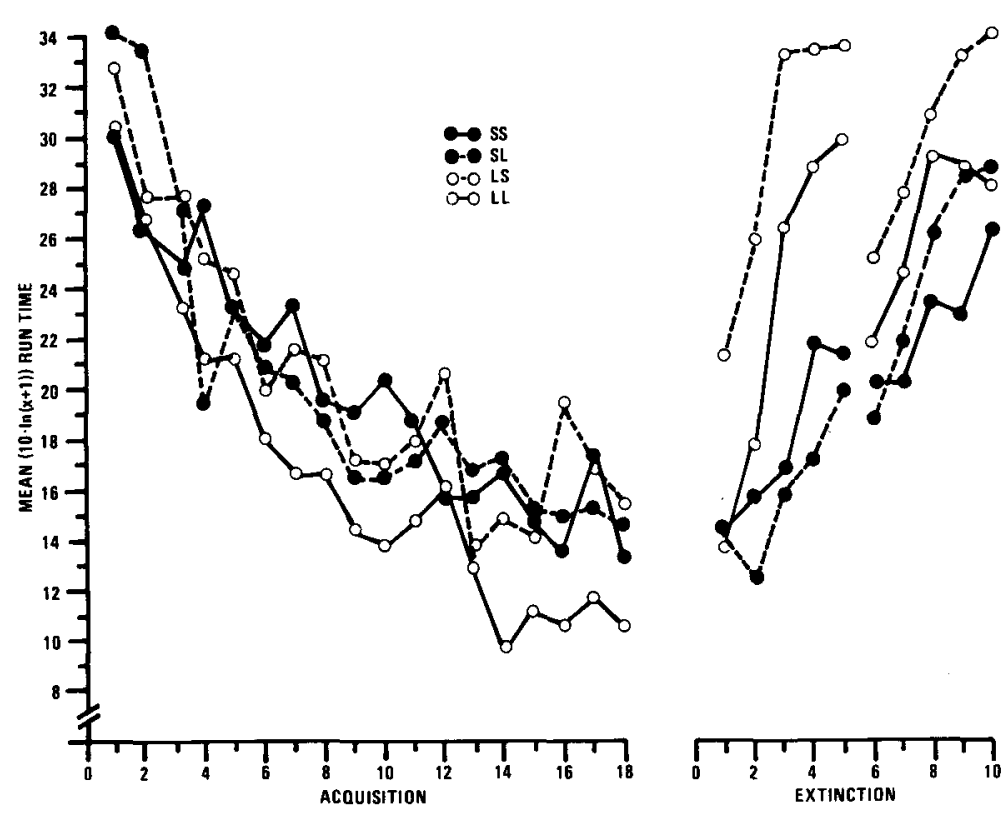

BLOCKS OF 2 TRIALS

effects did not persist into the second day of extinction $(F<1)$. The mean transformed run times on Day 1 of extinction for Groups LS, LL, SS, and SL were $29.49,23.35,18.00$, and 15.65 , respectively, each group differing significantly from the other except Groups SS and SL [1sd(27) $=5.25, \mathrm{p}<.05$ ]

Acquisition. Analysis of variance for acquisition treated the four groups as levels of a single between-subject factor (Groups), repeated within subjects over three blocks of 6 days with two trials within each day. Figure 2 depicts the significant $[F(2,54)=6.51, p<.01]$ interaction of Groups, Day Blocks, and Trials. The relationship of Groups SS and LL on Trials 1 and 2 remained relatively constant (LL running faster than SS) over each Day Block, suggesting that the significant Groups by Trials interaction, which developed over Day Blocks, was due primarily to Groups SL and LS. The varied magnitude groups not only ran fast on large-reward trials and slow on small-reward trials, but also ran slower on small-reward trials than the group given small reward on both trials (SS). A separate analysis on the last block of days showed that the animals were performing at a stable asymptotic level $[F(5,135)=1.14$, $p>.05]$ and that performance on the two daily trials differed between the groups $[F(3,27)=9.45, p<.01]$. Group SL ran significantly more slowly than Group SS on Trial $1[1 \mathrm{sd}(54)=5.55, \mathrm{p}<.05]$, but this simultaneous contrast effect was not significant in Group LS. Analysis of variance of the data relevant to the between-groups, amount-of-reward discrimination in the two consistently rewarded groups (LL and SS) showed that large reward produced faster running than small reward $[F(1,13)=4.90$, $\mathrm{p}<.05]$.

\section{Discussion}

Without exception, these results are in direct contradiction to what would be expected on the assumption that sucrose aftereffects are less effective, less persisting, and less discriminable than food-pellet aftereffects. The results, instead, clearly replicated Leonard's food-pellet findings. Small sucrose rewards on the first daily trial increased resistance to extinction relative to large rewards, and the opposite relation was true of reward magnitude on the second trial where small concentrations of sucrose decreased resistance to extinction relative to large. Further, the consistently rewarded groups showed an inverse

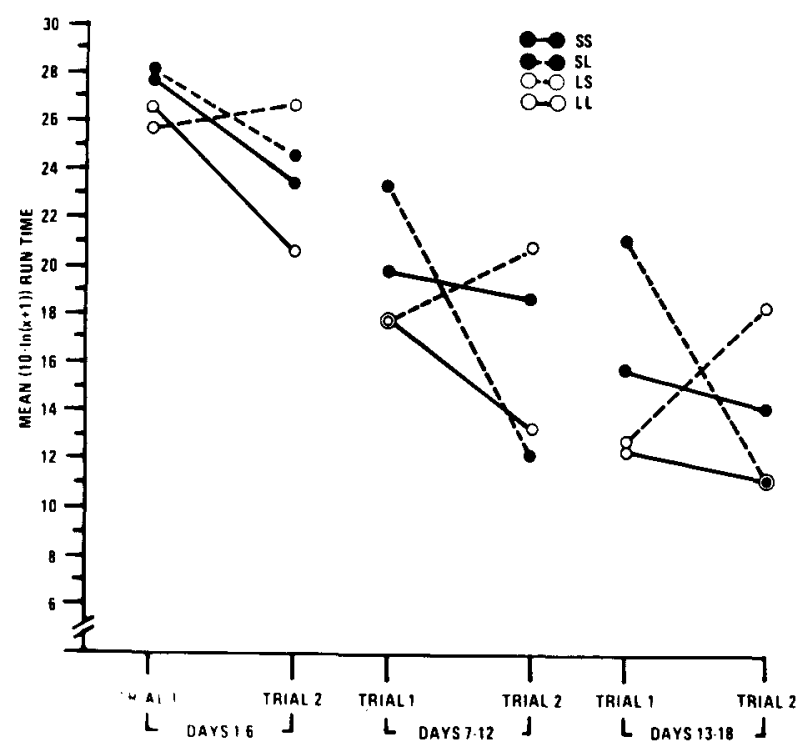

Figure 2. Mean transformed run time on each daily trial for the four groups of Experiment 1 shown in blocks of 6 days. 
relation between amount of reward and resistance to extinction. The simple view that sucrose rewards suffer from impoverished aftereffects is plainly inadequate. Sucrose rewards provided a potent source of aftereffect stimulation as evidenced by the extinction performance in this experiment. Some of the data from acquisition, in fact, suggest that sucrose may be an even better source of aftereffects than conventional solid food rewards.

Consider the fact that animals in the varied reward groups were patterning their responses in a manner appropriate for the reward on a given trial, a phenomenon deduced straightforwardly from aftereffect theory (Bloom, Williams, \& Metze, 1973; Capaldi, 1967). In other experiments of similar design using food pellets, no patterning was reported (Leonard, 1969; Leonard, Albin, \& Lebowitz, 1969).

The possibility that the patterning was governed by some learning of trial position per se but not aftereffects might be entertained since amount of reward and trial ordinal position were correlated in this experiment. It is difficult to see how this possibility could account for the ordering of groups in extinction, however, or how sucrose rewards might potentiate trial-position learning in the absence of aftereffect learning. On the other hand, if aftereffects were indeed controlling patterned responding, the fact that Group SL ran slower than Group SS on small reward trials, a simultaneous negative contrast effect (SiNCE), in this experiment with sucrose and not in previous food-pellet-reward experiments attests further to the saliency rather than the weakness of sucrose aftereffects. It now seems clear that the mechanisms of simultaneous and successive contrast are different, the most likely explanation of this difference involving the development of inhibition in the simultaneous discrimination (Burns, Woodard, Henderson, \& Bitterman, 1974; Gonzalez \& Powers, 1973; Mackintosh, 1974). While other simultaneous contrast experiments signal different reward magnitudes by external discriminative stimuli such as black or white runways (Bower, 1961), the stimuli in this study were, presumably, internal-the aftereffects of two different concentrations of sucrose reward.

\section{EXPERIMENT 2}

Several experiments (e.g., Franchina \& Sparling, 1973; Katz, Woods, \& Carrithers, 1966) have shown patterning in rats given single alternation (SA) of sucrose reward and nonreward with more than two trials/day, but their use of nonreward precludes assessment of the discriminability of the aftereffects of different sucrose magnitudes. SA of large and small solid food rewards has been studied only rarely (Bloom, Williams, \& Metze, 1973), but published accounts of SA with different sucrose magnitudes are nonexistent. An unpublished report by Likely
(Note 1) showed that differential leverpressing in rats did not develop during SA of $40 \%$ and $4 \%$ sucrose, a result which seems to conflict with the findings of Experiment 1 and, in fact, has been offered in support of the impoverished aftereffect view (Likely, Little, \& Mackintosh, 1971).

Experiment 2 was conducted to examine the runway performance of rats rewarded in a regularly alternating, extended sequence of large and small sucrose or food-pellet rewards.

\section{Method}

Subjects and Apparatus. The subjects were 18 experimentally naive male Long Evans rats, approximately 90 days old at the beginning of preliminary training. The apparatus was the same as that employed in Experiment 1, as were lighting conditions, arrangement of experimental equipment, etc.

Preliminary training. The rats were placed in individual cages in the experimental room, and, on Days 1-6 of preliminary training, were weighed daily and allowed $1 \mathrm{~h}$ on the walled exercise table. On Day 7, a food-deprivation schedule was initiated which was designed to reduce animals to $85 \%$ of their freefeeding body weights. During the subsequent 14 days, deprivation was continued as body weight adjusted to the new levels, feeding times were regulated to correspond with anticipated running times during the experiment proper, and all rats were allowed daily exercise on the table. Beginning on Day 22 , the animals were randomly divided into two groups of nine rats each. The group which was to receive sucrose rewards was placed on the table (in squads of four and five) and allowed $71 / 2 \mathrm{~min}$ access to $20 \%$ sucrose solution, delivered in the glass coaster, followed by $71 / 2 \mathrm{~min}$ access to $3 \%$ sucrose. The group which was to receive food pellets was allowed $15 \mathrm{~min}$ access (in squads of four and five) to a coaster kept full of $45-\mathrm{mg}$ Noyes pellets. The same exposure to reward was carried out on Day 23. On Day 24, onehalf of the rats from the sucrose group and one-half from the food group were given 10 goal placements with large $(1 \mathrm{ml}$ of $20 \%$ sucrose or 10 pellets) and small $(1 \mathrm{ml}$ of $3 \%$ sucrose or 1 pellet) reward of the appropriate type administered in a regularly alternating sequence beginning with large. The placement procedure was the same as in Experiment 1 . The remaining animals were given placements on Day 25.

Acquisition training. Animals were randomly divided into squads of two rats each and run in rotation to maintain an approximate 60-sec ITI. The reduction in ITI from Experiment 1 was to make the results more comparable to those of Bloom, Williams, and Metze (1973), who used a variable 15- or 45-sec ITI, and to those of Likely (Note 1), who used a 60-sec ITI. Rewards were the same as used in goal placements. During the first 4 days of acquisition training, four rats were dropped from the experiment either for failure to consume the reward on three successive trials or for failure to enter the goalbox within $5 \mathrm{~min}$ on three successive trials. This reduced the group sizes to seven rats each. Dummy trials were run to maintain the proper ITI in the two squads with only one rat each. After each squad was run, the animals were placed on the exercise table for $15 \mathrm{~min}$, then weighed, and fed in the home cage. The order of running of each squad was fixed to maintain approximately $23 \mathrm{~h}$ between feedings. Acquisition involved 10 trials each day with large and small reward alternating regularly (each day began with large). This training continued for 31 days. The running procedure on any particular trial was the same as in Experiment 1 .

\section{Results}

All time measures were transformed $[10 \times \ln (x+1)]$ for analysis. Although both start and run measures were sensitive to the experimental manipulations, 
only run times are presented here for brevity. The interaction between Reward Type, Reward Magnitude, and Day Blocks was significant $[\mathrm{F}(4,48)=3.77$, $\mathrm{p}<.01$ ], and is shown in Figure 3 .

On the first block of days, both food-pellet and sucrose animals ran faster for small reward than for large reward. This tendency persisted in the sucrose group through the third block of days, by which time the food-pellet group had begun to run fast on large-reward trials and slow on small-reward trials. Subsequent tests on simple main effects showed that reward magnitude was a significant factor on all but Day Blocks 2 and 3 for the foodpellet group, and was significant only on Day Block 1 for the sucrose group $[1 \mathrm{sd}(60)=1.15, \mathrm{p}<.05]$. Figure 3 also makes clear that the differentiation in response time in the food-pellet group came primarily through increases in the running time to small reward.

The significant interaction between Reward Type, Reward Magnitude, Day Blocks, and Trial Blocks $[F(16,192)=2.10, p<.01]$ reflects the different within-day development of the reward discrimination for both groups. Table 1 shows the differences in run times between the large- and small-reward trials within each daily session for food-pellet and sucrose groups averaged over the last two blocks of 6 days (during asymptotic acquisition performance). The measure shown in Table 1 was derived by subtracting the average Trial 1 running times (large reward) from the average Trial 2 running times (small reward) within each two-trial segment. The greatest discrimination between large and small reward

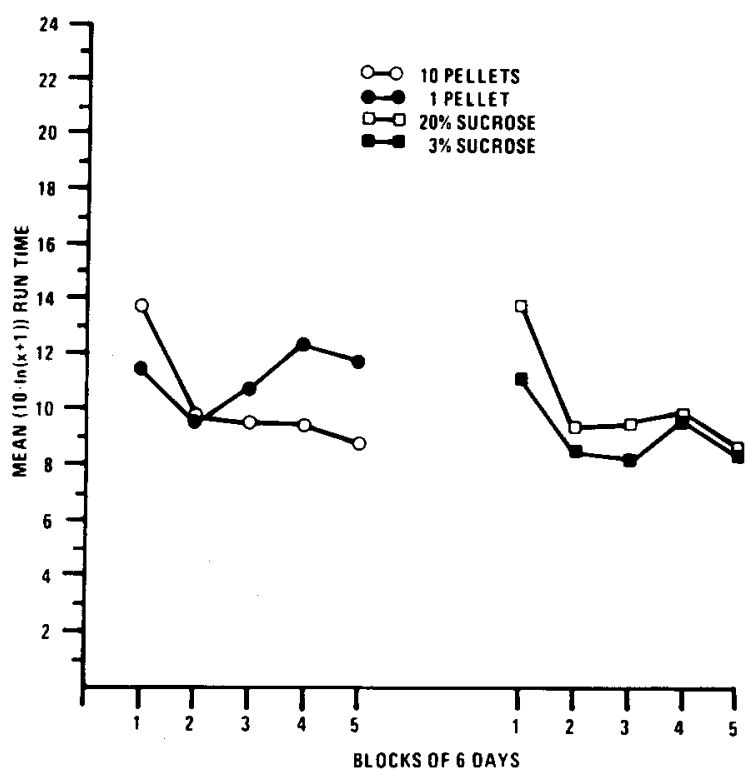

Figure 3. Mean transformed run time for food-pellet and sucrose groups on large and small reward trials in Experiment 2. Each point represents a mean of 6 days beginning with the second day of training. The sucrose group is shown to the right of the figure, food-pellet to the left.
Table 1

Difference Between Running Times for Large and Small Reward During Asymptotic Acquisition Performance: Experiment 2

\begin{tabular}{lrrrrr}
\hline & \multicolumn{5}{c}{ Trial Blocks } \\
\cline { 2 - 6 } \multicolumn{1}{c}{ Group } & \multicolumn{1}{c}{1} & 2 & 3 & \multicolumn{1}{c}{4} & 5 \\
\hline Food-pellet & 6.317 & 1.720 & 2.396 & 1.497 & 2.029 \\
Sucrose & -1.506 & -.267 & .643 & .259 & -.399 \\
\hline
\end{tabular}

occurred, for the food-pellet group, on the first two trials and reduced, generally, over the remaining trials. The same tendency is evident in the sucrose animals, except that the differences are in the reverse direction and are clearly smaller.

That reward magnitudes were adequately equated between food-pellet and sucrose rewards is evidenced by the insignificant main effect of Reward Type $(\mathrm{F}<1)$, the insignificant interaction between Reward Type and Day Blocks $(\mathrm{F}<1)$, and the insignificant effect of Reward Magnitude $(F<1)$.

\section{Discussion}

It is generally established in massed trials studies that patterned running is the result of the discriminated sensory consequences of the outcomes of preceding trials (Mackintosh, 1974; Tyler, Wortz, \& Bitterman, 1953), although issue has been taken with this view of the spaced-trials effect (e.g., Gonzalez \& Bitterman, 1969; Surridge \& Amsel, 1966). The conditioning of SRS by large reward should produce more habit strength $\left(\mathrm{S}^{\mathrm{RS}} \mathrm{H}_{\mathrm{R}}\right)$ for responses following small reward than the conditioning of SRL by small reward $\left(S^{R L} H_{R}\right)$ produces for responses following large reward. After some initial generalization, animals should begin to pattern appropriately. A modified view, proposed by Bloom, Williams, and Metze (1973) to account for patterning in discrete-trial leverpressing with large and small food-pellet rewards, assigns an inhibitory potential to the pairing of SRS with responses followed by large reward.

Experiment 2 provided additional evidence that SA of large and small food-pellet rewards produces patterning which is similar to the pattern observed with reward and nonreward (e.g., Flaherty \& Davenport, 1972). Further, this patterning was observed in a runway, extending the generality of the phenomenon. Running times on small reward trials, like the latencies in the Bloom et al. (1973) experiment, at first decreased (presumably due in part to generalization of $\mathrm{S}^{\mathrm{RS}} \mathrm{H}_{\mathrm{R}}$ ) then began to increase (due either to reduced generalization or to the accumulation of inhibition). But the compelling result of Experiment 2 is that sucrose-rewarded animals did not pattern as did those with food-pellet rewards and-with the same concentrations of sucrose-as they had done in Experiment 1. 


\section{GENERAL DISCUSSION}

Two procedural distinctions are apparent between Experiment 1 and Experiment 2. The ITI was reduced from 4 to $1 \mathrm{~min}$ and the number of daily trials was increased from 2 to 10 . That 2 daily trials might produce patterning more readily than 10 is suggested by the findings of Flaherty and Davenport (1972), which were substantiated by the results of Experiment 2. When 6 SA trials/day were administered in their study, the most distinctive patterning occurred during the first 2 daily trials, within-day discrimination reducing thereafter. Interestingly, the most likely interpretation of this effect is based upon a decrease, within days, of responsivity (Capaldi, 1967) produced by proactive interference. As daily training proceeds, the aftereffects on a given trial become less and less exclusive (low responsivity) due to confusion of the reward outcomes from preceding trials.

Krane and Wagner (1975) have reported that a .7\% saccharin solution is an ineffective Pavlovian CS when paired, in a trace conditioning paradigm, with an electric shock US at short but not at long CS-US intervals (the intervals ranged from 5 to $210 \mathrm{sec}$ ). This result suggests that the trace of saccharin is higher in amplitude and longer in duration than those of conventional exteroceptive CSs, lights and tones. The notion that sucrose aftereffects are strong and long-lasting relative to food-pellet aftereffects would explain why patterning with sucrose rewards was so readily obtained in Experiment 1 when the ITI was $4 \mathrm{~min}$ and not in Experiment 2 when it was 60 sec. Long traces might exceed short ITIs producing confused antecedents (low responsivity) on the subsequent trial. This view might also explain why patterning during acquisition was observed in Experiment 1 with sucrose and not in other experiments (Leonard, 1969; Leonard, Albin, \& Lebowitz, 1969) with the same ITI and the same number of daily trials but with food-pellet rewards. Finally, it would explain why food-pellet animals pattern in SA with 10 trials/day but sucrose animals do not. When only two trials/day are given with a relatively long ITI, the strong trace of sucrose rewards is more effective than the weaker trace of food-pellet rewards, but when more within-day trials are given at short ITIs, these same strong sucrose traces are less effective because they compound the interference from the outcomes of preceding trials.

If generalization decrement were the mechanism of the SuNCE, these findings would suggest that the inability to obtain SuNCEs with sucrose may in fact be due to low responsivity, but the low responsivity apparently comes from the persistence rather than the lability of sucrose aftereffects. To test the role of generalization decrement using the contrast design, concern should center on the effects of ITI length and the number of daily training trials as well as the interactions of these two variables with sucrose rewards. Generalization decrement with sucrose should be most apparent with two daily trials and a 4-min ITI.

The broader question of whether or not the absence of SuNCEs with goldfish and turtles, with changes in the delay of reward, and with sucrose instead of food-pellets as reward has a common explanation (Bitterman, 1975) is yet to be answered. It is interesting that rats trained with an immediate or 20-sec delayed food reward, 2 trials/day, show patterning and ordered extinction as did rats trained under similar conditions (except for the delays) with sucrose in Experiment 1 (Campbell, 1969). These delays, however, failed to produce patterning in SA with 6 trials/day (Horn \& Campbell, 1969) or with 10 trials/day (Cogan \& Capaldi, 1961). Goldfish do eventually pattern with SA of reward and nonreward (Gonzalez, 1972), but the effect is relatively difficult to obtain even with 2 trials/day (Mackintosh, 1971). There was no evidence, however, of ordered extinction in SL and LS groups when fish were trained in the Leonard design (Mackintosh, 1971). In situations involving relatively massed trials, it may well be that differences between fish and foodrewarded rats (in, for example, the Leonard design) are due to the fast decay rate of reward traces in fish, but similarities between fish and delayed or sucrose-rewarded rats-in the absence of further detailed investigations needed in this connection-do not seem to be explicable on the same grounds.

\section{REFERENCE NOTE}

1. Likely, D. Patterning of instrumental responding to sequences of varied food and sucrose rewards. Paper presented at meetings of the Eastern Psychological Association, Atlantic City, April 1970.

\section{REFERENCES}

Brtterman, M. E. The comparative analysis of learning. Science, 1975, 188, 699-707.

Bloom, J. M., Williams, D. T., \& Metze, L. D. Effects of varied and partial reward on discrete-trial patterning of rats. Animal Learning \& Behavior, 1973, 1, 167-170.

BOWER, G. H. A contrast effect in differential conditioning. Journal of Experimental Psychology, 1961, 62, 196-199.

Burns, R. A., Woodard, W. T., Henderson, T. B., \& Bitterman, M. E. Simultaneous contrast in the goldfish. Animal Learning \& Behavior, 1974, 2, 97-100.

CAmpbell, P. E. Sequences of delayed reward and extinction confinement: Effects on pattern running and extinction performance. Psychonomic Science, 1969, 1, 34-35.

CaPAldi, E. J. A sequential hypothesis of instrumental learning. In K. W. Spence \& J. T. Spence (Eds.), The psychology of learning and motivation (Vol. 1). New York: Academic Press, 1967.

CAPAldi, E. J., \& LynCh, A. D. Repeated shifts in reward magnitude: Evidence in favor of an associational and absolute (noncontextual) interpretation. Journal of Experimental Psychology, 1967, 75, 226-235.

Cogan, D., \& CAPAldi, E. J. Relative effects of delayed reinforcement and partial reinforcement on acquisition and extinction. 
Psychological Reports, 1961, 9, 7-13.

CRESPI, L. P. Quantitative variation of incentive performance in the white rat. American Journal of Psychology, 1942, 55, 467-517.

Elliotr, M. H. The effect of change of reward on the maze performance of rats. University of California Publications in Psychology, 1928, 4, 19-30.

Flaherty, C. F., \& Davenport, J. W. Successive brightness discrimination in rats following regular versus random intermittent reinforcement. Journal of Experimental Psychology, 1972, 96, 1.9.

Flaherty, C. F., Riley, E. P., \& SPear, N. E. Effects of sucrose concentration and goal units on runway behavior in the rat. Learning and Motivation, 1973, 4, 163-175.

Franchina, J. J., \& Brown, T. S. Reward magnitude shift effects in rats with hippocampal lesions. Journal of Comparative and Physiological Psychology, 1971, 76, 365-370.

Franchina, J. J., \& Sparling, D. L. Effects of sucrose concentrations on single alternation runway responding in rats. Learning and Motivation, 1973, 4, 471-479.

GonzALEZ, R. C. Patterning in goldfish as a function of magnitude of reinforcement. Psychonomic Science, 1972, 28, 53-55.

Gonzalez, R. C., \& Bitterman, M. E. Spaced-trials partial reinforcement as a function of contrast. Journal of Comparative and Physiological Psychology, 1969, 67, 94-103.

Gonzalez, R. C., Ferry, M., \& Powers, A. S. The adjustment of goldfish to reduction in magnitude of reward in massed trials. Animal Learning \& Behavior. 1974, 2, 23-26.

Gonzalez, R. C., Potts, A., Pitcoff, K., \& Bitterman, M. E. Runway performance of goldfish as a function of complete and incomplete reduction in amount of reward. Psychonomic Science, 1972, 27, 30S-307.

Gonzalez, R. C., \& Powers, A. S. Simultaneous contrast in goldfish. Animal Learning \& Behavior, 1973, 1, 96-98.

Homzie, M. J., \& Ross, L. E. Runway performance following a reduction in the concentration of a liquid reward. Journal of Comparative and Physiological Psychology, 1962, 55, 1029-1033.

Horn, J. A., \& Camprell, P. E. The effect of goal box delay and separate delay box delay on pattern running. Psychonomic Science, 1969, 17, 5-6.

Hulk, C. L. Principles of behavior. New York: Appleton-Century, 1943.

Hulc, C. L. A behavior system. New Haven: Yale University Press, 1952.

Katz, S., Woods, G., \& CarRithers, J. H. Reinforcement aftereffects and intertrial interval. Journal of Experimental Psychology, 1966, 72, 624-626.

KRANE, R. V., \& WAGNER, A. R. Taste aversion learning with a delayed shock US: Implications for the "generality of the laws of learning." Journal of Comparative and Physiological Psychology, 1975, 88, 882-889.

LEONARD, D. W. Amount and sequence of reward in partial and continuous reinforcement. Journal of Comparative and Physiological Psychology, 1969, 67, 204-211.

Leonard, D. W., Albin, R., \& Lebowitz, M. Performance under massed or spaced extinction following different sequences of varied reward training. Psychonomic Science, 1969, 16, 130-131.

Likely, D., Little, L., \& Mackintosh, N. J. Extinction as a function of magnitude and percentage of food or sucrose reward. Canadian Journal of Psychology Review of Canadian Psychology, 1971, 25, 130-137.

Lowes, G., \& Bitterman, M. E. Reward and learning in the goldfish. Science, $1967,157,455-457$.

Mackintosh, N. J. Reward and aftereffects of reward in the learning of goldfish. Joumal of Comparative and Physiological Psychology, 1971, 76, 225-232.

Mackintosh, N. J. The psychology of animal leaming. New York: Academic Press, 1974.

Mackintosh, N. J., \& LoRD, J. Simultaneous and successive contrast with delay of reward. Animal Learning \& Behavior, 1973, 1 , 283-286.

Pert, A., \& Bitterman, M. E. Reward and learning in the turtle. Learning and Motivation, 1970, 1, 121-128.

Pert, A., \& Gonzalez, R. C. Behavior of the turtle (Chrysemys picta picta) in simultaneous, successive, and behavioral contrast situations. Journal of Comparative and Physiological Psychology, 1974, 87, 526-538.

Rosen, A. J. Incentive-shift performance as a function of magnitude and number of sucrose rewards. Journal of Comparative and Physiological Psychology, 1966, 62, 487-490.

Rosen, A. J., Glass, D. H., \& Ison, J. R. Amobarbital sodium and instrumental performance changes following reward reduction. Psychonomic Science, 1967, 9, 129-130.

Rosen, A. J., \& Ison, J. R. Runway performance following changes in sucrose rewards. Psychonomic Science, 1965, 2, 335-336.

Shanab, M. E., France, J., \& Young, T. Negative contrast effect obtained with downshifts in magnitude but not concentration of solid sucrose reward. Bulletin of the Psychonomic Society, 1975. 5. 429-432.

Surridge, C. T., \& AMSel, A. Acquisition and extinction under single alternation and random partial-reinforcement conditions with a 24-hour intertrial interval. Journal of Experimental Psychology, 1966, 72, 361-368.

Tyler, D. W., Wortz, E. C., \& Bitterman, M. E. The effect of random and alternating partial reinforcement on resistance to extinction in the rat. American Journal of Psychology, 1953, 66, $57-65$.

\section{NOTE}

1. The list of categories probably exceeds those mentioned by Mackintosh (1974), including the use of certain barbiturate drugs (Rosen, Glass, \& Ison, 1967) as well as hippocampal lesions (Franchina \& Brown, 1971).

(Received for publication May 14, 1976; revision accepted August 9, 1976.) 